\title{
WASP-16b: A NEW JUPITER-LIKE PLANET TRANSITING A SOUTHERN SOLAR ANALOG
}

\author{
T. A. Lister ${ }^{1}$, D. R. Anderson ${ }^{2}$, M. Gillon ${ }^{3,4}$, L. HebB ${ }^{5}$, B. S. Smalley ${ }^{2}$, A. H. M. J. Triaud ${ }^{3}$, A. Collier Cameron ${ }^{5}$,

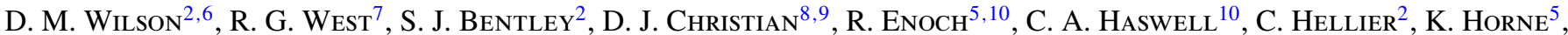

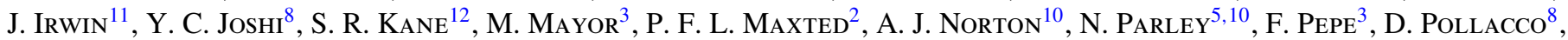 \\ D. Queloz ${ }^{3}$, R. Ryans ${ }^{8}$, D. Segransan ${ }^{3}$, I. Skillen ${ }^{13}$, R. A. Street ${ }^{1}$, I. Todd ${ }^{8}$, S. Udry ${ }^{3}$, and P. J. Wheatley ${ }^{14}$ \\ ${ }^{1}$ Las Cumbres Observatory, 6740 Cortona Drive Suite 102, Goleta, CA 93117, USA; tlister@1cogt.net \\ 2 Astrophysics Group, Keele University, Staffordshire, ST5 5BG, UK \\ ${ }^{3}$ Observatoire de Genève, Universit' de Genève, 51 Ch. des Maillettes, 1290 Sauverny, Switzerland \\ ${ }^{4}$ Institut d'Astrophysique et de Géophysique, Université de Liège, Allée du 6 Août, 17, Bat. B5C, Liège 1, Belgium \\ ${ }^{5}$ SUPA, School of Physics and Astronomy, University of St Andrews, North Haugh, St Andrews, Fife KY16 9SS, UK \\ ${ }^{6}$ Centre for Astrophysics \& Planetary Science, School of Physical Sciences, University of Kent, Canterbury, Kent, CT2 7NH, UK \\ ${ }^{7}$ Department of Physics and Astronomy, University of Leicester, Leicester, LE1 7RH, UK \\ ${ }^{8}$ Astrophysics Research Centre, School of Mathematics \& Physics, Queen's University, University Road, Belfast, BT7 1NN, UK \\ ${ }^{9}$ California State University, 18111 Nordhoff Street, Northridge, CA 91330-8268, USA \\ ${ }^{10}$ Department of Physics and Astronomy, The Open University, Milton Keynes, MK7 6AA, UK \\ ${ }^{11}$ Harvard-Smithsonian Center for Astrophysics, 60 Garden Street, Cambridge, MA 02138, USA \\ ${ }^{12}$ NASA Exoplanet Science Institute, Caltech, MS 100-22, 770 South Wilson Avenue, Pasadena, CA 91125, USA \\ ${ }^{13}$ Isaac Newton Group of Telescopes, Apartado de Correos 321, E-38700 Santa Cruz de la Palma, Tenerife, Spain \\ ${ }^{14}$ Department of Physics, University of Warwick, Coventry, CV4 7AL, UK \\ Received 2009 April 24; accepted 2009 August 4; published 2009 September 1
}

\begin{abstract}
We report the discovery from WASP-South of a new Jupiter-like extrasolar planet, WASP-16b, which transits its solar analog host star every 3.12 days. Analysis of the transit photometry and radial velocity spectroscopic data leads to a planet with $R_{\mathrm{p}}=1.008 \pm 0.071 R_{\mathrm{Jup}}$ and $M_{\mathrm{p}}=0.855 \pm 0.059 M_{\mathrm{Jup}}$, orbiting a host star with $R_{*}=0.946 \pm 0.054 R_{\odot}$ and $M_{*}=1.022 \pm 0.101 M_{\odot}$. Comparison of the high resolution stellar spectrum with synthetic spectra and stellar evolution models indicates the host star is a near-solar metallicity $([\mathrm{Fe} / \mathrm{H}]=$ $0.01 \pm 0.10)$ solar analog $\left(T_{\text {eff }}=5700 \pm 150 \mathrm{~K}\right.$ and $\left.\log g=4.5 \pm 0.2\right)$ of intermediate age $\left(\tau=2.3_{-2.2}^{+5.8} \mathrm{Gyr}\right)$.
\end{abstract}

Key words: planetary systems - stars: abundances - stars: individual (WASP-16b)

\section{INTRODUCTION}

There are currently over 300 known exoplanets ${ }^{15}$ with the majority of them discovered through the radial velocity (RV) technique. A growing number of exoplanets in recent years have been discovered through the transit method. Transiting exoplanets are particularly valuable as they allow parameters such as the mass, radius, and density to be accurately determined and further studies such as transmission spectroscopy, secondary eclipse measurements, and transit timing variations to be carried out.

There are several wide angle surveys that have been successful in finding transiting exoplanets around bright stars, namely HAT (Bakos et al. 2002), TrES (Alonso et al. 2004), XO (McCullough et al. 2005), and WASP (Pollacco et al. 2006). The WASP Consortium conducts the only exoplanet search currently operating in both hemispheres although HATnet is planning a southern extension and several groups are planning searches from Antarctica (e.g., Strassmeier et al. 2007; Crouzet et al. 2009).

We report the discovery from the WASP-South observatory of a $\sim 0.86 M_{\text {Jup }}$ mass companion orbiting a $V \sim 11.3$ close solar analog WASP-16 (=TYC 6147-229-1, USNO-B1.0 06970298329).

\section{OBSERVATIONS}

\subsection{Photometric Observations}

WASP-South, located at SAAO, South Africa, is one of two SuperWASP instruments and comprises eight cameras on a

\footnotetext{
15 http://exoplanet.eu
}

robotic mount. Each camera consists of a Canon $200 \mathrm{~mm} f / 1.8$ lens with an Andor $2048 \times 2048 \mathrm{e} 2 \mathrm{v}$ CCD camera giving a field of view of $7.8 \times 7.8$ and a pixel scale of 13".7. Exposure times were $30 \mathrm{~s}$ and the same field is returned to and reimaged every $8-10$ minutes. Further details of the instrument, survey, and data reduction pipelines are given in Pollacco et al. (2006); and the candidate selection procedure is described in Collier Cameron et al. (2007), Pollacco et al. (2008), and references therein.

WASP-16 was observed for a partial season in 2006, a full season in 2007, and a further partial season in 2008 with the distribution of data points as 3324 points (2006), 6013 (2007), and 4084 (2008). The 2007 light curve revealed the presence of a $\sim 1.3 \%$ dip with a period of $\sim 3.11$ days. The transit coverage in the other two seasons was very sparse, particularly in 2006, and there is only evidence for two partial transits in the 2008 data. WASP-16 was a fairly strong candidate for follow up despite the small number of transits, passing the filtering tests of Collier Cameron et al. (2006) with a signal to red noise ratio, $S_{\text {red }}=9.38$ (with $S_{\text {red }}>5$ required for selection), "transit to antitransit ratio" $\Delta \chi^{2} / \Delta \chi^{2}{ }_{-}=2.5\left(\Delta \chi^{2} / \Delta \chi^{2}{ }_{-} \geqslant 1.5\right.$ required for selection) and no measurable ellipsoidal variation.

The SuperWASP light curve showing a zoom of the transit region, along with the model transit fit, is shown in Figure 1. In order to better constrain the transit parameters, followup high precision photometric observations with the Swiss $1.2 \mathrm{~m}+$ EULERCAM on La Silla, were obtained in the $I_{c}$ band on the night of 2008 May 4 and are shown in Figure 2.

\subsection{Spectroscopic Observations}

In order to confirm the planetary nature of the transit signal, we obtained follow-up spectroscopic observation with the 


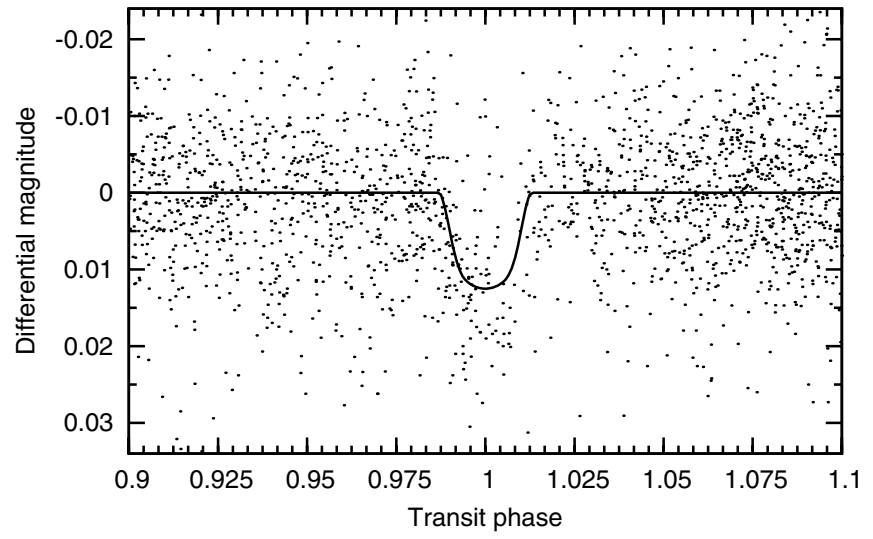

Figure 1. Zoom of the transit region of the SuperWASP light curve of WASP$16 \mathrm{~b}$ with the best-fitting MCMC model overplotted.

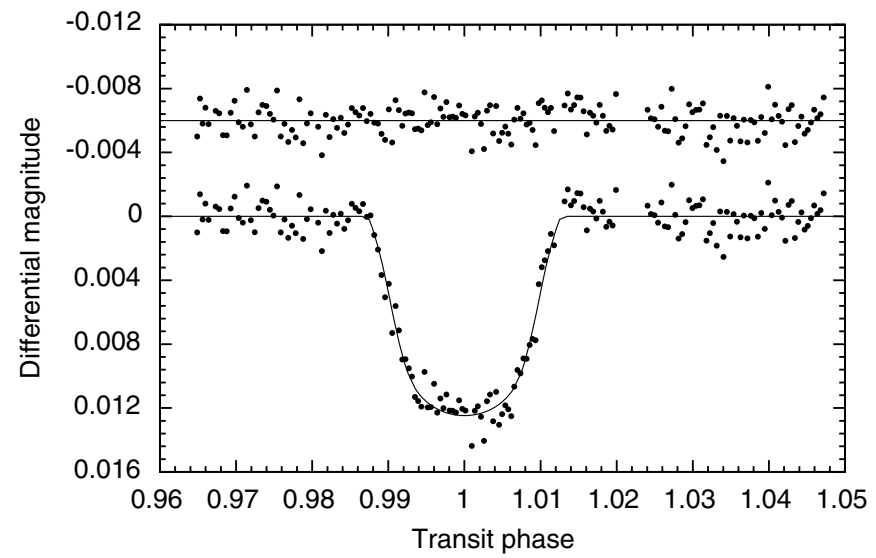

Figure 2. $I_{C}$ band light curve from EULERCAM and residuals from the transit fit of WASP-16b.

Swiss $1.2 \mathrm{~m}+$ CORALIE spectrograph. The data were processed through the standard CORALIE reduction pipeline as described by Baranne et al. (1996) with an additional correction for the blaze function. Fourteen RV measurements were made between 2008 March 10 and 2008 August 4 and an additional sixteen between 2009 February 19 and 2009 June 3 (see Table 1) by crosscorrelating with a G2 template mask. The resulting RV curve is shown in Figure 3. The low amplitude RV variation clearly supports the existence of a planetary mass companion. In order to rule out a nonplanetary explanation for the RV variation such as a blended eclipsing binary or starspots, we examined the line-bisector spans. Contamination from an unresolved eclipsing binary will cause asymmetries in the spectral line profiles and line-bisector span variations (Queloz et al. 2001; Torres et al. 2005). As can be seen from the lower panel of Figure 3, there is no sign of variation with phase of the bisector spans and their amplitude is much smaller than the RV variation. This supports the conclusion that the $\mathrm{RV}$ variations are due to a planet orbiting the star and not due to some other cause.

\section{WASP-16 SYSTEM PARAMETERS}

\subsection{Stellar Parameters}

The individual CORALIE spectra are of relatively low signalto-noise ratio $(\mathrm{S} / \mathrm{N})$, but when coadded into $0.01 \AA$ steps they give a $\mathrm{S} / \mathrm{N}$ of around 70:1 which is suitable for a photospheric analysis of the host star. In addition, a single HARPS spectrum was used to complement the CORALIE analysis, but this
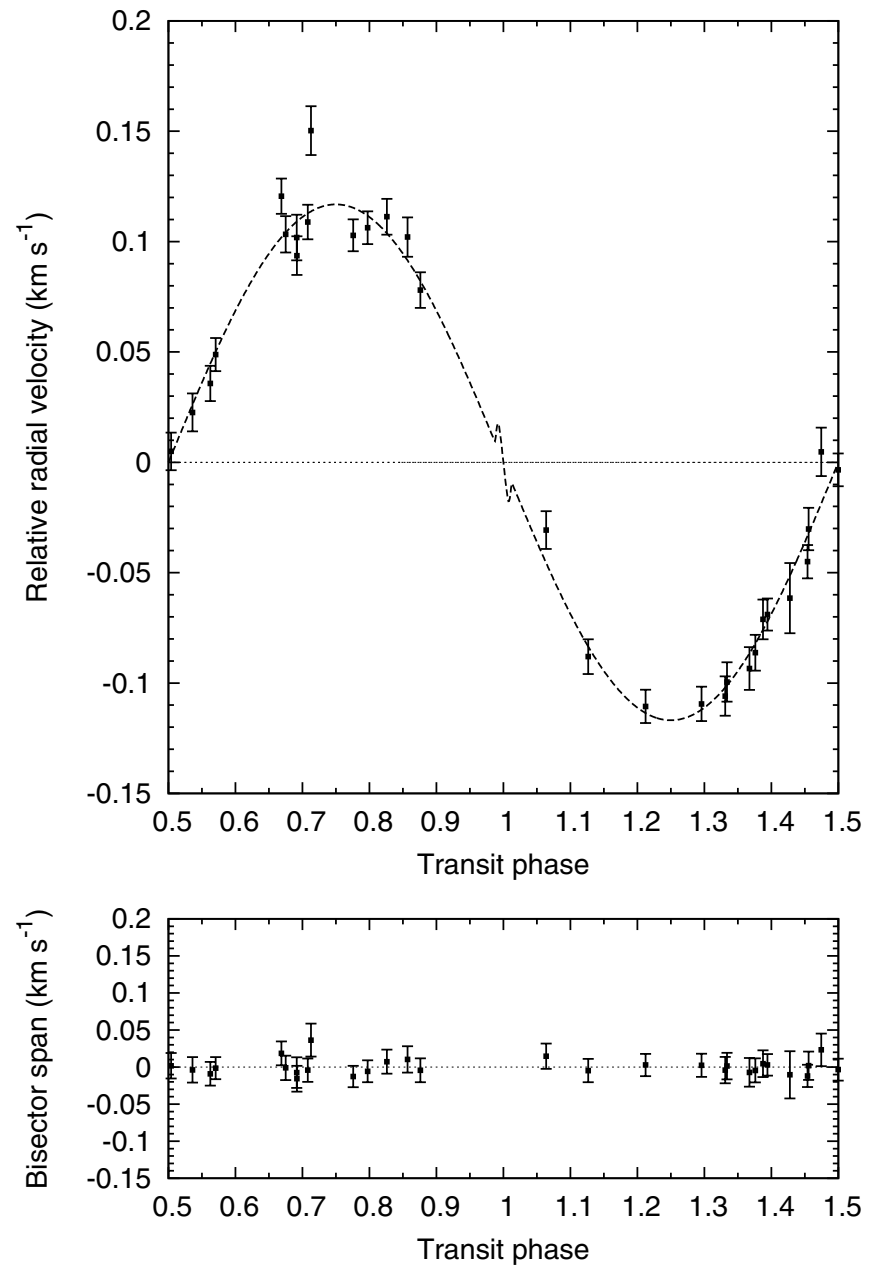

Figure 3. Radial velocity curve (upper panel) of WASP-16 from the Swiss $1.2 \mathrm{~m}+\mathrm{CORALIE}$ along with the best-fitting model which includes the predicted Rossiter-McLaughlin effect. The resulting bisector spans are shown in the lower panel. The uncertainties on the bisector spans are double the radial velocity uncertainties.

spectrum had relatively modest $\mathrm{S} / \mathrm{N}$ of around 50:1. The standard CORALIE/HARPS pipeline reduction products were used in the analysis.

The analysis was performed in a very similar fashion to that described by West et al. (2009) using a spectral synthesis package and LTE model atmospheres. The $H_{\alpha}$ and $H_{\beta}$ lines were used to determine the effective temperature $\left(T_{\text {eff }}\right)$, while the Na I D and $\mathrm{Mg}$ I b lines were used as surface gravity $(\log g)$ diagnostics. In addition, the $\mathrm{Ca} \mathrm{H}$ and $\mathrm{K}$ lines provided a further check on the derived $T_{\text {eff }}$ and $\log g$. The elemental abundances of several elements were determined from measurements of several clean and unblended lines. The parameters and the abundances obtained from the analysis are listed in Table 2 .

In our spectra, the Li I $6708 \AA$ line is not detected (EW $<2 \mathrm{~m} \AA$ ), allowing us to derive an upper limit on the Lithium abundance of $\log n(\mathrm{Li} / \mathrm{H})+12<0.8$. The lack of lithium would imply an age in excess of $5 \mathrm{Gyr}$ (Sestito \& Randich 2005). The stellar rotation velocity $(v \sin i)$ was determined by fitting the profiles of several $\mathrm{Fe}$ I lines using an average value of $v_{\mathrm{mac}}=$ $2.0 \mathrm{~km} \mathrm{~s}^{-1}$ for the macroturbulence $\left(v_{\mathrm{mac}}\right)$.

In addition to the spectral analysis, we have also used available broadband photometry to estimate the total observed bolometric flux. The Infrared Flux Method (Blackwell \& Shallis 1977) was then used with Two Micron All Sky Survey 
Table 1

CORALIE Radial Velocities for WASP-16

\begin{tabular}{|c|c|c|c|}
\hline $\begin{array}{l}\text { Time of Obs. } \\
\text { (BJD-2450000) }\end{array}$ & $\begin{array}{l}\text { Rad. Vel. } \\
\left(\mathrm{km} \mathrm{s}^{-1}\right)\end{array}$ & $\begin{array}{c}\sigma_{\mathrm{RV}} \\
\left(\mathrm{km} \mathrm{s}^{-1}\right)\end{array}$ & $\begin{array}{c}\text { Bisector Span } \\
\left(\mathrm{km} \mathrm{s}^{-1}\right)\end{array}$ \\
\hline 4535.864842 & -1.99772 & 0.01591 & 0.00306 \\
\hline 4537.849158 & -1.96688 & 0.00853 & -0.04553 \\
\hline 4538.858364 & -2.00734 & 0.00899 & -0.03129 \\
\hline 4558.780835 & -1.83336 & 0.00723 & -0.02779 \\
\hline 4560.709473 & -2.00513 & 0.00725 & -0.02403 \\
\hline 4561.688137 & -1.82730 & 0.00785 & -0.03998 \\
\hline 4589.705102 & -1.84255 & 0.00875 & -0.04520 \\
\hline 4591.706755 & -2.03571 & 0.00892 & -0.03221 \\
\hline 4652.495906 & -1.82493 & 0.00808 & -0.03209 \\
\hline 4656.551645 & -2.02421 & 0.00787 & -0.02555 \\
\hline 4657.577293 & -1.96640 & 0.00957 & -0.01827 \\
\hline 4663.539741 & -2.02961 & 0.00969 & -0.02661 \\
\hline 4664.616769 & -1.78590 & 0.01108 & -0.04350 \\
\hline 4682.521501 & -1.98118 & 0.00754 & -0.02123 \\
\hline 4881.869213 & -2.02245 & 0.00813 & -0.02760 \\
\hline 4882.801025 & -1.83289 & 0.00823 & -0.03739 \\
\hline 4884.737094 & -2.04565 & 0.00778 & -0.01672 \\
\hline 4891.805707 & -1.90043 & 0.00798 & -0.01009 \\
\hline 4892.723980 & -1.83413 & 0.00891 & -0.02116 \\
\hline 4941.728231 & -1.88737 & 0.00748 & -0.04134 \\
\hline 4943.730102 & -2.04677 & 0.00753 & -0.01825 \\
\hline 4944.739293 & -1.91359 & 0.00860 & -0.02245 \\
\hline 4945.799895 & -1.85815 & 0.00807 & 0.01502 \\
\hline 4947.745317 & -1.93960 & 0.00741 & -0.03134 \\
\hline 4948.673112 & -1.82992 & 0.00743 & -0.06231 \\
\hline 4972.707323 & -1.93123 & 0.00854 & -0.03631 \\
\hline 4975.733486 & -1.93144 & 0.01100 & -0.01416 \\
\hline 4982.647535 & -1.83433 & 0.01036 & -0.02677 \\
\hline 4984.642389 & -2.04210 & 0.00892 & -0.04270 \\
\hline 4985.694776 & -1.81561 & 0.00802 & -0.02406 \\
\hline
\end{tabular}

(2MASS) magnitudes to determine $T_{\text {eff }}$ and stellar angular diameter $(\theta)$. This gives $T_{\text {eff }}=5550 \pm 130 \mathrm{~K}$, which is in close agreement with that obtained from the spectroscopic analysis $\left(T_{\text {eff }}=5700 \pm 150 \mathrm{~K}\right)$.

Comparison with the stellar evolution models of Girardi et al. (2000) for solar metallicity $(Z=0.02)$ gives maximumlikelihood values $M_{*}=1.00_{-0.067}^{+0.045} M_{\odot}$ as shown in Figure 4 . Alternative models from Baraffe et al. (1998) give essentially the same results as the stellar evolution models have close agreement in this mass range. The uncertainties on the stellar
Table 2

Stellar Parameters of the WASP-16 Host Star

\begin{tabular}{lc}
\hline \hline Parameter & Value \\
\hline R.A. & $14^{\mathrm{h}} 18^{\mathrm{m}} 43^{\mathrm{s}} .92$ \\
Decl. & $-20^{\circ} 16^{\prime} 31^{\prime \prime} .8(\mathrm{~J} 2000.0)$ \\
$T_{\text {eff }}$ & $5700 \pm 150 \mathrm{~K}$ \\
$\log g$ & $4.5 \pm 0.2$ \\
$\xi_{\mathrm{t}}$ & $1.1 \pm 0.2 \mathrm{~km} \mathrm{~s}^{-1}$ \\
$v \sin i$ & $3.0 \pm 1.0 \mathrm{~km} \mathrm{~s}^{-1}$ \\
$\mathrm{Spectral} \mathrm{type}$ & $\mathrm{G} 3 \mathrm{~V}^{\mathrm{a}}$ \\
{$[\mathrm{Fe} / \mathrm{H}]$} & $0.01 \pm 0.10$ \\
{$[\mathrm{Na} / \mathrm{H}]$} & $0.15 \pm 0.08$ \\
{$[\mathrm{Mg} / \mathrm{H}]$} & $0.14 \pm 0.10$ \\
{$[\mathrm{Si} / \mathrm{H}]$} & $0.10 \pm 0.07$ \\
{$[\mathrm{Ca} / \mathrm{H}]$} & $0.11 \pm 0.12$ \\
{$[\mathrm{Sc} / \mathrm{H}]$} & $0.14 \pm 0.07$ \\
{$[\mathrm{Ti} / \mathrm{H}]$} & $0.05 \pm 0.14$ \\
{$[\mathrm{~V} / \mathrm{H}]$} & $0.09 \pm 0.15$ \\
{$[\mathrm{Cr} / \mathrm{H}]$} & $0.02 \pm 0.11$ \\
{$[\mathrm{Co} / \mathrm{H}]$} & $0.17 \pm 0.08$ \\
{$[\mathrm{Ni} / \mathrm{H}]$} & $0.07 \pm 0.12$ \\
$\log A(\mathrm{Li})$ & $<0.8$ \\
$T_{\text {eff }}(\mathrm{IRFM})$ & $5550 \pm 130 \mathrm{~K}$ \\
$\theta(\mathrm{IRFM})$ & $0.052 \pm 0.003 \mathrm{mas}$ \\
&
\end{tabular}

Note.

${ }^{\text {a }}$ Estimated from $J-H$ color

density lead to a large uncertainty on the age from the Girardi et al. (2000) isochrones producing an estimated age of $\tau=$ $2.3_{-2.2}^{+5.8}$ Gyr.

\subsection{Planet Parameters}

The CORALIE spectroscopic RV data were combined with the WASP-South and EULERCAM photometric data in a simultaneous fit to determine the planetary parameters. The method of Markov Chain Monte Carlo (MCMC) as detailed in previous investigations (Pollacco et al. 2008; Collier Cameron et al. 2007) was used. We used the Claret (2000) limb darkening coefficients for the appropriate stellar temperature and photometric passband and a adaptive stepsize mechanism is used during the 5000 step burn-in phase until the chain converges. At the end of this phase, the adaptive stepsize mechanism is switched off for the final 20,000 steps in the chain. The autocorrelation length

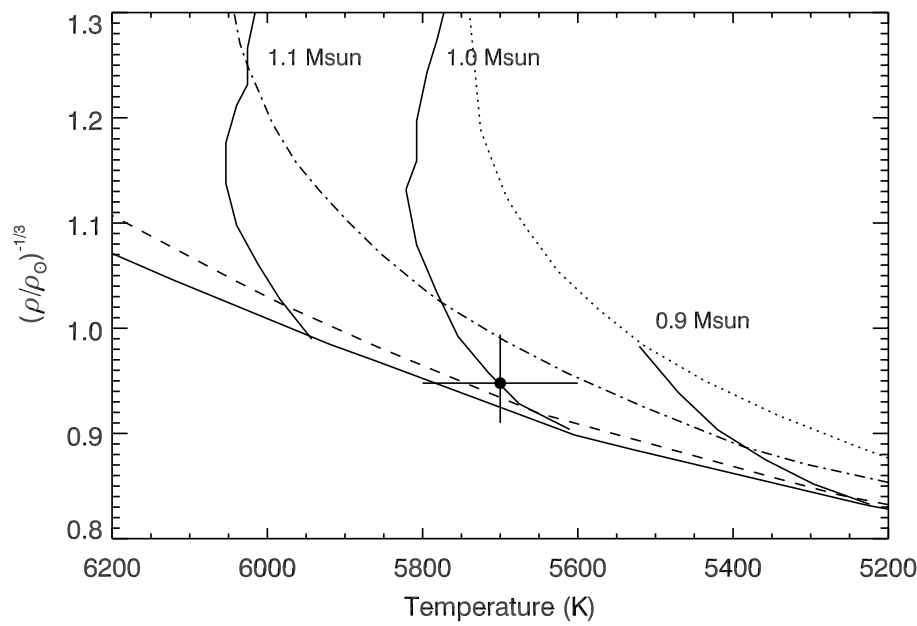

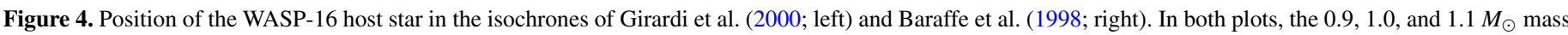
tracks are shown along with $100 \mathrm{Myr}$ (solid), 1 Gyr (dashed), 5 Gyr (dot-dashed), and 10 Gyr (dotted) isochrones. 
Table 3

System Parameters for WASP-16b

\begin{tabular}{|c|c|c|}
\hline Parameter & Value & Error \\
\hline$P$ (days) & 3.1186009 & $\begin{array}{l}+0.0000146 \\
-0.0000131\end{array}$ \\
\hline$T_{0}(\mathrm{HJD})$ & 2454584.42878 & $\begin{array}{l}+0.00035 \\
-0.00023\end{array}$ \\
\hline$T_{\text {dur }}$ (days) & 0.0800 & $\begin{array}{l}+0.0018 \\
-0.0012\end{array}$ \\
\hline$R_{\mathrm{P}}^{2} / R_{*}^{2}$ & 0.01199 & $\begin{array}{l}+0.00052 \\
-0.00039\end{array}$ \\
\hline$b \equiv a \cos i / R_{*}$ & 0.798 & $\begin{array}{l}+0.026 \\
-0.019\end{array}$ \\
\hline$e$ & \multicolumn{2}{|c|}{0 (adopted) } \\
\hline$K_{1}\left(\mathrm{~km} \mathrm{~s}^{-1}\right)$ & 0.1167 & $\begin{array}{l}+0.0024 \\
-0.0019\end{array}$ \\
\hline$\gamma\left(\mathrm{km} \mathrm{s}^{-1}\right)$ & -1.93619 & $\begin{array}{l}+0.00021 \\
-0.00023\end{array}$ \\
\hline$a(\mathrm{AU})$ & 0.0421 & $\begin{array}{l}+0.0010 \\
-0.0018\end{array}$ \\
\hline$i(\operatorname{deg})$ & 85.22 & $\begin{array}{l}+0.27 \\
-0.43\end{array}$ \\
\hline$M_{*}\left(M_{\odot}\right)$ & 1.022 & $\begin{array}{l}+0.074 \\
-0.129\end{array}$ \\
\hline$R_{*}\left(R_{\odot}\right)$ & 0.946 & $\begin{array}{l}+0.057 \\
-0.052\end{array}$ \\
\hline $\log g_{*}(\mathrm{cgs})$ & 4.495 & $\begin{array}{l}+0.030 \\
-0.054\end{array}$ \\
\hline$\rho_{*}\left(\rho_{\odot}\right)$ & 1.21 & $\begin{array}{l}+0.13 \\
-0.18\end{array}$ \\
\hline$M_{\mathrm{p}}\left(M_{\mathrm{Jup}}\right)$ & 0.855 & $\begin{array}{l}+0.043 \\
-0.076\end{array}$ \\
\hline$R_{\mathrm{p}}\left(R_{\mathrm{Jup}}\right)$ & 1.008 & $\begin{array}{l}+0.083 \\
-0.060\end{array}$ \\
\hline$\rho_{\mathrm{p}}\left(\rho_{\text {Jup }}\right)$ & 0.83 & $\begin{array}{l}+0.13 \\
-0.17\end{array}$ \\
\hline $\log g_{\mathrm{p}}(\mathrm{cgs})$ & 3.284 & $\begin{array}{l}+0.041 \\
-0.064\end{array}$ \\
\hline$T_{\mathrm{eq}}(A=0, \quad F=1)(\mathrm{K})$ & 1280 & $\begin{array}{l}+35 \\
-21\end{array}$ \\
\hline Safronov number $(\Theta)$ & 0.070 & \pm 0.010 \\
\hline
\end{tabular}

of the chain was $9 \pm 1$ for all the parameters, indicating that no unwanted correlations are present and the chain is "well mixed."

Initial fits showed that the eccentricity was poorly constrained but consistent with zero and so was fixed at this value in subsequent fits. The prior on the stellar mass was set to $1.0 M_{\odot}$, as indicated by the evolutionary tracks discussed in the previous section, but no constraint or prior on the stellar radius or density was used in the fit. The transit parameters such as the period, depth, and duration were initially set at the values from the transit search of the WASP-South data and subsequently refined in the MCMC code using all the available data.

The best-fitting system parameters are listed in Table 3 and show that WASP-16b is a reasonably close Jupiter analog albeit somewhat less massive and in a $P \sim 3$ day orbit. The host star has a fitted mass and radius which are slightly smaller than the Sun, leading to a slightly higher density than the solar case; but all the parameters are identical to the Sun within the error bars. The lack of lithium detection, low $v \sin i$ and similar large inferred age also point toward WASP-16 being a solar analog hosting a hot Jupiter planet.

\section{TIMES OF TRANSIT}

Although WASP-16 was observed with WASP-South for one full and two partial seasons, there are very few complete transits within the timeseries suitable for determining times of transits. This illustrates the need for long timeseries on potential transit fields as shown by Smith et al. (2006). In total, we find four complete and well-measured transits from the SuperWASP data and these are shown in Table 4 as "Fitted $T_{0}$ " along with one time of transit determined from the EULERCAM data. The predicted times of transit from the MCMC ephemeris (given in Table 3) are also shown in Table 4 . There are currently an insufficient number of measured transits with adequate precision to suggest anything other than a constant period.
Table 4

Times of Transit for WASP-16b

\begin{tabular}{lccr}
\hline \hline $\begin{array}{c}\text { Fitted } T_{0} \\
(\text { HD-2450000) }\end{array}$ & $\begin{array}{c}\text { Error } \\
(\text { days })\end{array}$ & $\begin{array}{c}\text { Predicted } T_{0} \\
(\text { HJD-2450000) }\end{array}$ & $\begin{array}{r}\text { O-C } \\
(\text { days })\end{array}$ \\
\hline 4216.43288 & 0.00243 & 4216.43417 & -0.00129 \\
4238.26861 & 0.00458 & 4238.26436 & 0.00425 \\
4266.31992 & 0.06319 & 4266.33175 & -0.01183 \\
4291.27823 & 0.03342 & 4291.28054 & -0.00231 \\
4590.66606 & 0.00028 & 4590.66602 & 0.00004 \\
\hline
\end{tabular}

\section{CONCLUSIONS}

We report the discovery of a new transiting planet with the WASP-South station of the SuperWASP survey. The planet, designated WASP-16b, orbits a star which is a close solar analog, having temperature, mass, radius, metallicity, and gravity the same as the Sun, within the error bounds. The age of the host star, with an admittedly large error bar, is also close to the solar age.

The orbiting planet is a reasonable Jupiter analog although somewhat less massive than Jupiter $\left(M_{p} \sim 0.85 M_{\text {Jup }}\right)$, but with a near identical radius $\left(R_{p} \sim 1.01 R_{\text {Jup }}\right)$ leading to a density some $80 \%$ of Jupiter. This planet falls in the lower left corner of the group of "normal" Jupiter-sized planets in the mass/radius diagram, with the majority of objects in this region being somewhat larger than Jupiter in either mass or radius. Additionally, if we compute the Safronov number $\Theta \equiv \frac{1}{2}\left(V_{\text {esc }} / V_{\text {orb }}\right)^{2}=0.070 \pm 0.010$ for this planet, this along with its equilibrium temperature $T_{\mathrm{eq}}=1280 \mathrm{~K}$ places it in the center of the Class I planets as defined by Hansen \& Barman (2007). The "normality" of this planet makes it similar to WASP$2 \mathrm{~b}, \mathrm{TrES}-1 \mathrm{~b}$, and other "normal" extrasolar planets and stands in contrast to the inflated radii and low densities of planets like TrES-4b (Sozzetti et al. 2009), HD 209458b (Brown et al. 2001), and WASP-1b (Collier Cameron et al. 2007).

The WASP Consortium comprises the Universities of Keele, Leicester, St. Andrews, the Queen's University Belfast, the Open University, and the Isaac Newton Group. WASP-South is hosted by the South African Astronomical Observatory, and we are grateful for their support and assistance. Funding for WASP comes from the consortium universities and from the UK's Science and Technology Facilities Council.

Facilities: Euler1.2m, ESO:3.6m

\section{REFERENCES}

Alonso, R., et al. 2004, ApJ, 613, L153

Bakos, G. Á., Lázár, J., Papp, I., Sári, P., \& Green, E. M. 2002, PASP, 114, 974 Baraffe, I., Chabrier, G., Allard, F., \& Hauschildt, P. H. 1998, A\&A, 337, 403 Baranne, A., et al. 1996, A\&AS, 119, 373

Blackwell, D. E., \& Shallis, M. J. 1977, MNRAS, 180, 177

Brown, T. M., Charbonneau, D., Gilliland, R. L., Noyes, R. W., \& Burrows, A 2001, ApJ, 552, 699

Claret, A. 2000, A\&A, 363, 1081

Collier Cameron, A., et al. 2006, MNRAS, 373, 799

Collier Cameron, A., et al. 2007, MNRAS, 375, 951

Crouzet, N., et al. 2009, in Proc. of IAU Symp. 253, Transiting Planets, ed. F. Pont, D. Queloz, \& D. Sasselov (Cambridge: Cambridge Univ. Press), 336 Girardi, L., Bressan, A., Bertelli, G., \& Chiosi, C. 2000, A\&AS, 141, 371

Hansen, B. M. S., \& Barman, T. 2007, ApJ, 671, 861

McCullough, P. R., Stys, J. E., Valenti, J. A., Fleming, S. W., Janes, K. A., \& Heasley, J. N. 2005, PASP, 117, 783

Pollacco, D. L., et al. 2006, PASP, 118, 1407

Pollacco, D., et al. 2008, MNRAS, 385, 1576

Queloz, D., et al. 2001, A\&A, 379, 279 
Sestito, P., \& Randich, S. 2005, A\&A, 442, 615

Smith, A. M. S., et al. 2006, MNRAS, 373, 1151

Sozzetti, A., et al. 2009, ApJ, 691, 1145

Strassmeier, K. G., Andersen, M. I., Granzer, T., Korhonen, H., Herber, A., Cutispoto, G., Rafanelli, P., \& Horne, K. 2007, in ASP Conf. Ser. 366,
Transiting Extrapolar Planets Workshop, ed. C. Afonso, D. Weldrake, \& T. Henning (San Francisco, CA: ASP), 332

Torres, G., Konacki, M., Sasselov, D. D., \& Jha, S. 2005, ApJ, 619, 558

West, R. G., et al. 2009, AJ, 137, 4834 